\title{
Negative Index Materials: New Frontiers in Optics
}

\section{Costas M. Soukoulis ${ }^{1,2}$, Maria Kafesaki ${ }^{2}$, and E. N. Economou ${ }^{3}$}

${ }^{1}$ Department of Physics and Ames Laboratory, Iowa State University, Ames, Iowa, USA

${ }^{2}$ IESL-FORTH, P.O. Box 1527, 71110 Heraklion, Crete Greece, and Department of Materials Science and Technology, University of Crete, Heraklion, Greece

${ }^{3}$ IESL-FORTH, and Department of Physics, University of Crete, Heraklion, Crete, Greece

\begin{abstract}
Recently a lot of interest has been focused on a new class of materials, the so-called lefthanded materials (LHMs) or negative index materials (NIMs) that exhibit highly unusual electromagnetic properties and promise new device applications. These materials do not exist in nature and can only be fabricated artificially, and for this reason are called metamaterials. Their unique properties are not determined by the fundamental physical properties of their constituents but by the shape and the distribution of the specific patterns
\end{abstract}


included in them. Metamaterials can be designed to exhibit both electric and magnetic resonances that can be separately tuned to occur in frequency bands from $\mathrm{MHz}$ to $\mathrm{THz}$ frequencies, and hopefully to the visible. This article presents a short history of the field, describes the underlying physics, and reviews the experimental and theoretical present status of the field. Many interesting questions on how to fabricate more isotropic LHMs, on how to push the operational frequency to optical wavelengths, how to reduce the losses, and how to incorporate active or nonlinear materials in LHMs, remain to be explored. 


\section{Introduction}

\section{History of LHMs}

Electromagnetic metamaterials are artificially structured media with unique and distinct properties that are not observed in naturally occurring materials. More than three decades ago Victor Veselago [1] predicted many unusual properties of a fictitious (at that time) isotropic medium with simultaneously negative electrical permittivity $(\varepsilon)$ and magnetic permeability $(\mu)$, which he named left-handed material (LHM). As Veselago showed, LHMs will display unique "reversed" electromagnetic properties, as a result of an electromagnetic wave in such a medium having the triad $\mathbf{k}, \mathbf{E}, \mathbf{H}$ left handed and, hence, exhibiting phase and group velocities of opposite directions. It follows also that a LHM is characterized by a negative refractive index. The latter leads, in particular, to Cherenkov radiation, Doppler shift, radiation pressure, and even Snell's law being reversed in LHMs. These revolutionary properties open up a new regime in physics and technology (e.g. almost zero reflectivity at any angle of incidence), provided that such LHMs can be realized.

Veselago's initial suggestion remained completely hypothetical since the naturally occurring materials do not provide such properties, until a significant breakthrough was announced in 2000: Smith et. al. [2,3], presented evidence for a composite medium - interlaced lattices of conducting rings and wires (see Fig. 1(a)) - displaying negative values for $\varepsilon$ and $\mu$. 


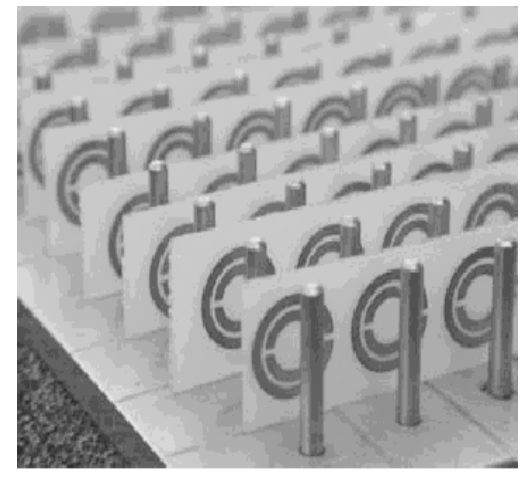

(a)

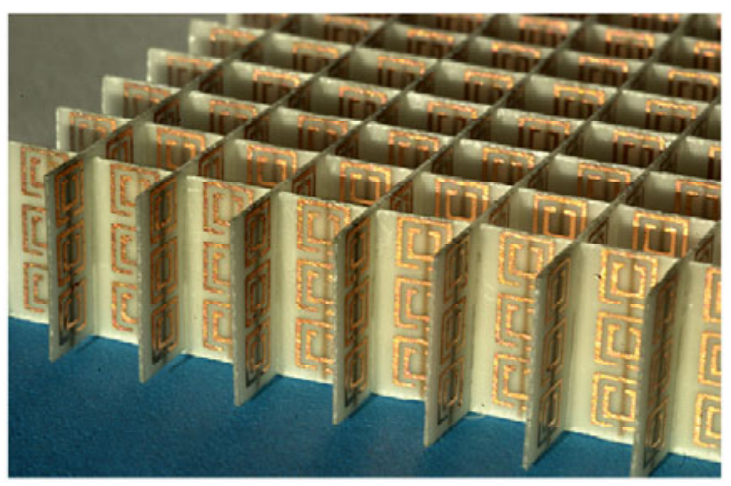

(b)

Fig. 1: (a) The composite Left-Handed (LH) medium employed in Ref. 2.The medium consists of Split Ring Resonators (SRR), created lithographically on a circuit board material, and metallic posts. (b) A split ring structure etched into circuit board plus copper wires to give negative $\mu$ and negative $\varepsilon$. Taken from Ref. 3

For the development of the first LHM, David Smith and his colleagues followed the pioneering work of J. Pendry, who, in 1999, developed designs [4] for structures that are magnetically active although made of non-magnetic materials. One of those structures is the so-called split-ring resonator (SRR) structure, composed of metallic rings with gaps (see Fig. 1(a)), which has been widely adopted as the model for creating negative $\mu$ at $\mathrm{GHz}$ frequencies. The SRR structure has proven a remarkably efficient means of producing a magnetic response and has been recently scaled down in size (and thus upwards in frequency) to produce metamaterials active at $\mathrm{THz}$ frequencies (see discussion below). Pendry and his colleagues have applied related to the SRR designs to Magnetic Resonance Imaging (MRI) studies, in the MHz region of the spectrum [5].

A key property of a LHM is that it possesses a negative index of refraction-a novel and remarkable material property. One of the most dramatic - and controversial- prediction of the LHMs was that by Pendry [6] which stated that a thin negative-index film should behave as a "superlens", providing image detail with a resolution beyond the diffraction limit, to which all positive-index lenses are subject. Conventional positive-refractive index lenses require curved surfaces to bend the rays 
emanating from an object to form an image. Yet, Pendry and Veselago noted that negative refractive index lenses are not subject to the same constraint; they found that a planar slab of material with a refractive index of -1 could also produce an image. For this lens, diverging rays from a nearby object are negatively refracted at the first surface of the slab, reversing their trajectory so as to converge at a focus within the material (see Fig. 2). The rays diverge from this focus and are again negatively refracted at the second surface, finally converging to form a second image just outside the slab. Although it produces an image, the planar lens differs from conventional curved-surface lenses in that it does not focus parallel rays, and has a magnification that is always unity.

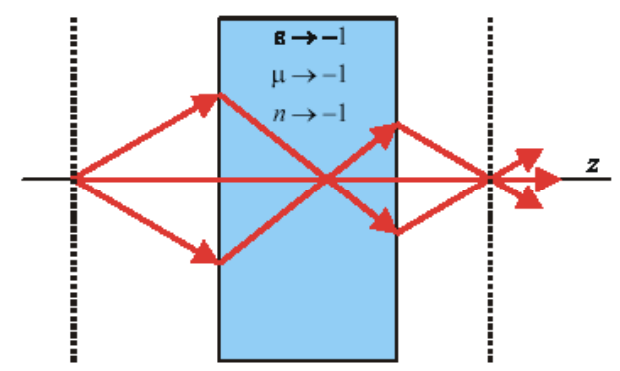

Fig. 2. A negative refractive index medium bends light to a negative angle relative to the surface normal. Light formerly diverging from a point source in the object plane is reversed and converges back to a point. Released from the medium the light reaches a focus for a second time in the image plane.

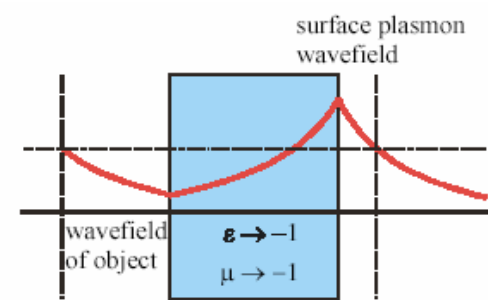

Fig. 3. The principle of evanescent wave refocusing. The exponentially decaying component of the wave from the object on the left (which is responsible for the diffraction limited resolution) grows exponentially within the planar $n=-1$ lens. The new lens works by excitation of surface plasmons. Matching the fields at the boundaries selectively excites a surface plasmon on the far surface thus reproducing the same amplitude in the image plane as in the object plane.

On careful reexamination of this planar lens, Pendry found that it is, in principle, capable to recover also the evanescent waves emanating from an object. (The electromagnetic field of an object includes not only propagating waves, but also near-field "evanescent" waves that decay exponentially as a function of the distance away from the object.) These evanescent waves carry the 
finest details of the object, but their recovery by conventional positive-index lenses is minimal and only at the very near field, which leads to a resolution no better than roughly one-half of the illuminating wavelength - the diffraction limit. Pendry found that in a planar negative-index lens an evanescent wave decaying away from an object grows exponentially in the lens; on exiting the lens, the wave decays again until it reaches the image plane, where it has the same amplitude with which it started (see Fig. 3). Unlike any other lens, the resolution limit of the planar negative-index lens is determined by how many evanescent waves from the object can be recovered, rather by the diffraction limit (in practice several stringent requirements limit the perfect focusing). The radical nature of the beating of the diffraction limit rose many objections, initially suggesting that this is impossible $[7,8]$.
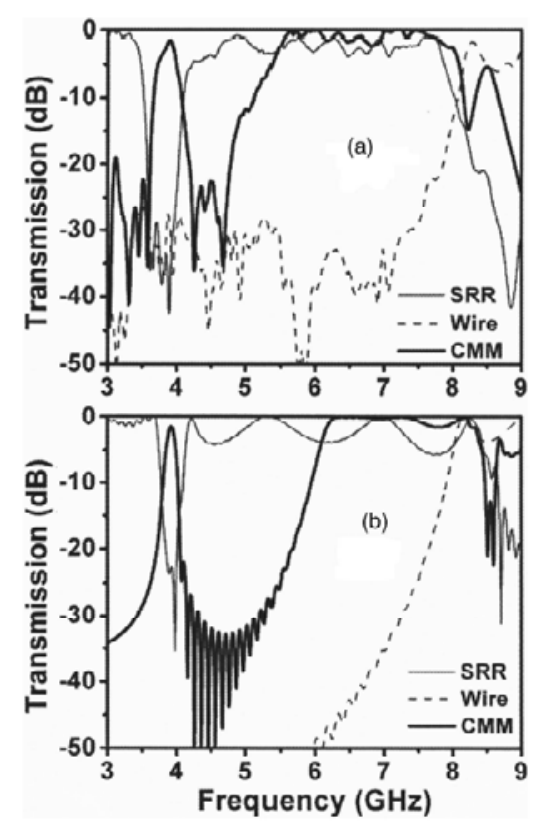

Fig. 4. Transmission spectra of SRRs, wires, and Composite Meta-Materials (CMM), i.e. SRRs \& wires: (a)

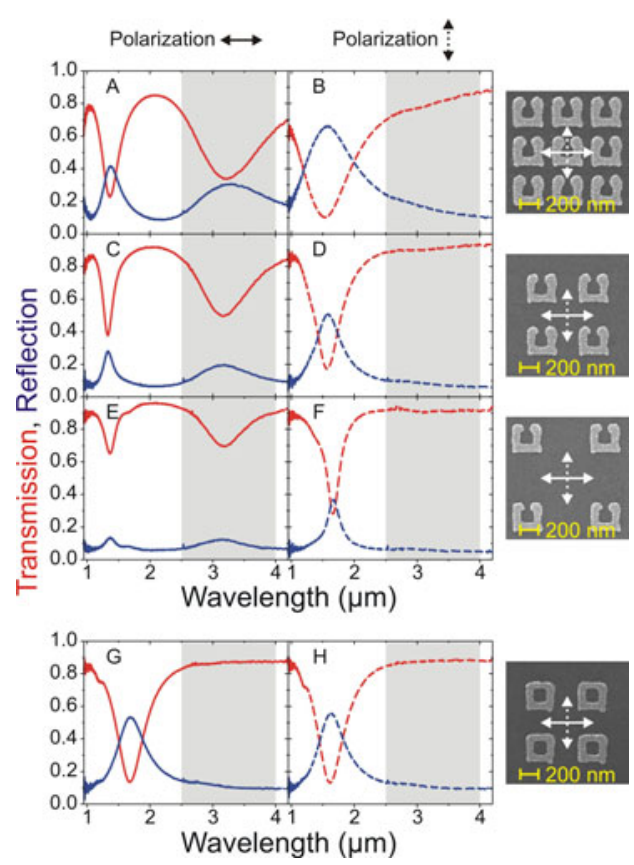

Fig. 5. Measured transmission (red) and reflection (blue) spectra. In each row of this "matrix", an electron micrograph of the sample is shown on the RHS. The two polarization configuration 
In the last four years (see Refs. 7 and 8 for recent reviews of the LHM field) different groups, by careful experiments, sophisticated simulations and physical analysis, have come up with new, optimized structures [9-18], mostly at microwave frequencies (see Fig. 4, where the best transmission left-handed peak is shown [14]; losses are only $-0.3 \mathrm{~dB} / \mathrm{cm}$ ). These structures exhibit unambiguously negative index of refraction; therefore the widespread acceptance of the existence of Negative Index of refraction Materials (NIMs) is well established.

Recently, many groups have fabricated samples and observed [25-30] negative $\mu$ at the $\mathrm{THz}$ region. In Figure 5 we present results for SRRs which give a negative $\mu$ at $100 \mathrm{THz}$ [26]. The Karlsruhe group manages to fabricate [28] SRRs which give a negative $\mu$ at $200 \mathrm{THz}$ ! This is an amazing accomplishment for the LHMs field. No negative $n$ is seen yet at $100 \mathrm{GHz}$ and THz region. Thus, it is a great challenge of this field to be able to combine the SRR structures, which give negative $\mu$, with the wires, that give negative $\varepsilon$, to produce a negative index material at $\mathrm{THz}$ and optical frequencies. However, as one moves to optical frequencies the losses of the metallic elements of the LHMs might be a problem and need to be addressed in detail. 


\section{Negative refraction in photonic crystals}

A different approach to achieve negative index of refraction [31-34, 22-24] is to use photonic crystals (PCs). The PCs can be made from only dielectrics and can, in principle, have much less losses than the metallic LHMs, especially at high frequencies, and even at the optical range. In PCs, to achieve negative

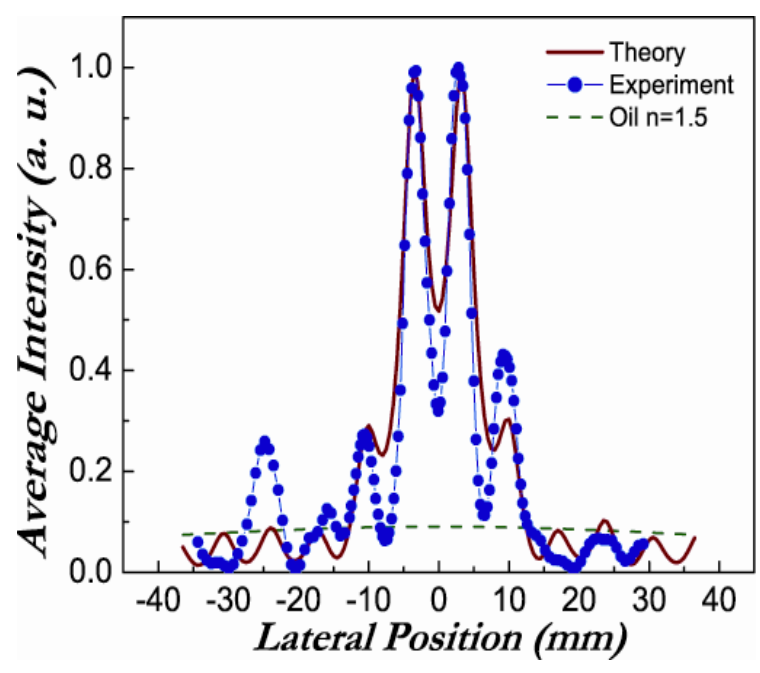

\footnotetext{
Fig. 6. Measured power distribution (solid point) and calculated average intensity (solid line) at $0.7 \mathrm{~mm}$ away from the second interface of a photonic crystal for two incoherent sources at a distance $\lambda / 3$. So sub-wavelength resolution was achieved. The wavelength $\lambda=22 \mathrm{~mm}$. Calculated average intensities at these points with dielectric slabs are also shown (dashed line). Taken from Ref. 23.
}

refraction, the size and the periodicity of the "atoms" (elementary units) should be of the order of the wavelength. In regular LHMs the size of the unit cell is much smaller than the wavelength and therefore the effective medium theory can be applied and one is able to define effective values of $\varepsilon$ and $\mu$. In PCs no effective $\varepsilon$ and $\mu$ can be defined, although the phase and group velocity are reversed, as in regular LHMs. One has to be very careful in distinguishing the LH behavior from Bragg scattering effects [34].

Both negative refraction and left-handed behavior have been demonstrated in PCs [22-24]. Cubukcu et. al. used dielectric rods to show negative refraction [22] as well as focusing and superlensing [23] in PCs 
at microwave frequencies. One of their results is shown in Fig. 6, where one can see subwavelength resolution obtained from a photonic crystal consisting of a square array of dielectric rods in air (rods dielectric constant is $\varepsilon=9.61$, diameter $2 r=3.15 \mathrm{~mm}$, length $l=15 \mathrm{~cm}$ and lattice constant $a=4.79$ $\mathrm{mm})$. The same structure was used to demonstrate also negative refraction in photonic crystals [22]. Negative refraction and focusing in PCs in the microwave regime has been also demonstrated by Parini et. al. [24], using metallic PCs. Parini et al. [24], as well as Parazolli et. al. [35] used a planoconcave lens fabricated from a PC and a LHM, respectively, to demonstrate focusing of a plane wave. An inverse experiment $[24,35]$, in which a plane wave is produced from a point source placed at the focal point of the lens was also performed. While most of the experiments in PCs [22-24] were performed at microwave frequencies, the same structures scaled at optical frequencies must have much less losses than the LHMs, which are based on metallic elements. Nevertheless, only two experimental demonstrations of negative refraction in the near IR frequency region have been made so far in PCs (in PCs made of GaAs [36] and Si-polymide [37]).

\section{Cut-wires and negative index of refraction}

Recent theoretical work [38-40] concerning attempts to achieve magnetic response from metallic elements has shown that pairs of finite in length (short) wires would not only be able to replace the SRRs, but possibly could also lead to a negative refractive index directly, without the need of additional metallic wires. The condition to obtain simultaneously negative $\varepsilon$ and $\mu$ by pairs of finite metallic wires is very restrictive. Recent experiments [41-43] have shown evidence of negative $n$ at $\mathrm{THz}$ frequencies, employing finite wires. The observed negative $n$ though was most probably due to the significant imaginary parts of $\varepsilon$ and $\mu$ [44], which lead also to a dominant imaginary part of $n$ and thus to a rapid attenuation of electromagnetic (EM) waves, which makes such kind of meta-materials inapplicable. 


\section{Polaritonic photonic crystals}

An alternative approach to fabricate metamaterials that would have both $\varepsilon$ and $\mu$, and therefore $n$, negative is to use PCs composed of polaritonic materials [45-48]. O'Brien and Pendry [46] have shown that that a $2 \mathrm{D}$ square $\mathrm{PC}$ of circular ferroelectric rods has a resonance in $\mu$ in the millimeter wavelength range. Huang et. al. [47] found that a 2D PC composed of polaritonic materials behaves as an effective medium with negative permeability in the micron wavelength range. The resonance in $\mu$ in such a medium is due to the large values of $\varepsilon(\omega)$ attained near the transverse phonon [47] frequency, $\omega_{T}$. Shvets [48] has suggested that the metallic behavior $(\varepsilon<0)$ of polaritonic materials above $\omega_{T}$, combined with a PC, that gives $\mu<0$, can be used to construct LHMs with $\varepsilon$ and $\mu$ simultaneously negative. There are no experiments demonstrating these very interesting ideas yet.

\section{Nonlinear LHMs}

While most of the work on LHMs has been done in the linear regime, where both the magnetic permeability and the dielectric permittivity are assumed to be independent of the intensity of the EM field, Kivshar and his colleagues [49-50] were the first to study the nonlinear properties of LHMs, with some very interesting results. The combination of NIMs with other active or nonlinear materials is a very interesting area of research, which needs to be pursued. It might lead to new NIMs with reduced losses at the optical wavelengths, broader bandwidth and other features not possible with passive NIMs. Likewise, the combination of NIMs with various types of nonlinear materials will result in nonlinear LHMs, which have already been predicted to offer new and unusual properties, such as soliton formation, second harmonic generation, bistability, phase conjugation and phase matching. Finally, methods to switch and

modulate LHMs can possibly be implemented by combining LHMs with other materials whose EM parameters can be dynamically tuned by the application of external electric or magnetic fields. 


\section{Electric and magnetic response of metamaterials}

As we discussed in the previous section, the first experimental materialization of Pendry's ideas was made by Smith et. al. in 2000 [2], and since then various new samples were prepared (composed of SRRs and wires) all of which have been shown to exhibit a pass band in which it was assumed that $\varepsilon$ and $\mu$ are both negative. This assumption was based on measuring independently the transmission, $T$, of the wires alone, and then the $T$ of the SRRs alone. If the peak in the combined metamaterial composed of SRRs+wires were in the stop bands for the SRRs alone (which corresponds to negative $\mu$ ) and for the wires alone (which is thought to correspond to negative $\varepsilon$ ) the peak was considered to be left-handed (LH). Further support to this interpretation was provided by the demonstration that some of these materials exhibit negative refraction of electromagnetic waves [3].
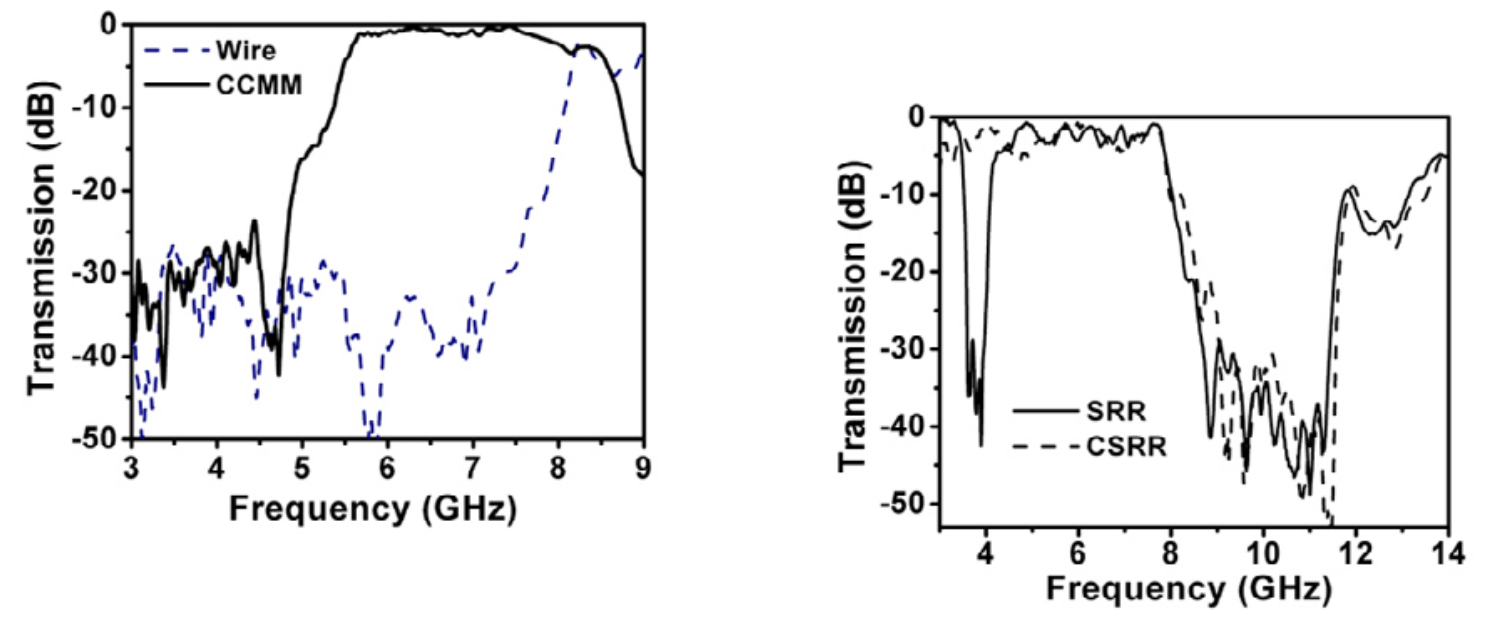

Fig. 7. Measured transmission spectra of a lattice of

Fig. 8. Measured transmission spectra of a periodic arrangement of SRRs (solid line) and closed SRRs 
wires (dashed line) and a closed CMM (solid line) composed of closed SRRs (CSRRs) and wires. Notice that the plasma frequency (corresponding to the onset of this dip is magnetic in origin. significant transmission) of the closed $\mathrm{CMM}$ is at 5 $\mathrm{GHz}$, and is much lower that the plasma frequency of wires only, which is at $8 \mathrm{GHz}$. (dashed line). Notice that the transmission dip at $4 \mathrm{GHz}$ disappears by the closing of the gaps of the SRRs. So

Subsequent experiments [19] have reaffirmed the property of negative refraction, giving strong support to the interpretation that these metamaterials can be correctly described by negative permeability, due to the SRRs, and negative permittivity, due to the wires. However, as was shown in Ref. 51, this is not always the case, as the SRRs exhibit also a resonant electric response, in addition to their magnetic response, which was first described by Pendry [4] and will be analyzed in the last part of this section. The electric response of the SRRs, which is demonstrated by closing their air gaps (destroying therefore their magnetic response), is identical to that of cut-wires, and it is added to the electric response $(\varepsilon)$ of the wires. The result is the effective plasma frequency, $\omega_{\mathrm{p}}{ }^{\prime}$, of the combined system of wires and SRRs (or closed SRRs) to be always lower than the plasma frequency of only the wires, $\omega_{\mathrm{p}}$ (see Fig. 7). In Fig. 8, one clearly sees that by closing the gaps of the SRRs the transmission dip at $\sim 4 \mathrm{GHz}$ disappears, while the rest of the spectrum remains almost unchanged. This shows that the $\sim 4 \mathrm{GHz}$ dip is magnetic in origin and it is due to the LC character of the SRR (see next paragraph), and also that the closing of the SRR gaps does not affect all the other aspects of its response (the last is valid only if SRR has mirror symmetry in respect to the incident electric field). With this consideration and the analytical expressions for $\varepsilon$ and $\mu$ [51], which stem from it, one is able to reproduce all the low frequency transmission, $T$, and reflection, $R$, characteristics of LHMs. Even the minor details in $T$ and $R$ observed in the simulations can be analytically explained. Moreover, an easy criterion to identify if an experimental transmission peak is LH or righthanded (RH) can be achieved: If the closing of the gaps of the SRRs in a given LHM structure removes 
the peak close to the position of the SRR dip from the $T$ spectrum, this is a strong evidence that the $T$ peak is indeed left-handed. If the gap above the peak is removed, the peak is most likely right-handed. This criterion is very valuable in experimental studies [14], where one cannot easily obtain the effective $\varepsilon$ and $\mu$. The criterion is used experimentally and is found that some $T$ peaks that were thought to be LH turned out to be right-handed [13].

It is well known from elementary electromagnetism (EM) that a magnetic dipole can be realized by the circulating current of a closed metallic loop, like a closed SRR (CSRR), which leads to a magnetic moment, $m$, with magnitude given by the product of the current and the area of the CSRR, and direction perpendicular to the plane of the SRR. Therefore, CSRR behaves as an inductor, storing magnetic energy $U=m B=L I^{2} / 2$, where $L$ is the self-inductance of the loop. If a CSRR is combined with a capacitor, with capacitance $C$, then one obtains an LC circuit, with a resonance frequency $\omega_{L C}=1 / \sqrt{L C}$. Such a capacitor can be realized by making a cut in the ring, leading to a normal SRR. Thus, SRR acts like an electromagnetic resonator, producing at $\omega_{L C}$ resonant circular currents, leading to resonant magnetization, i.e. resonant effective permeability.

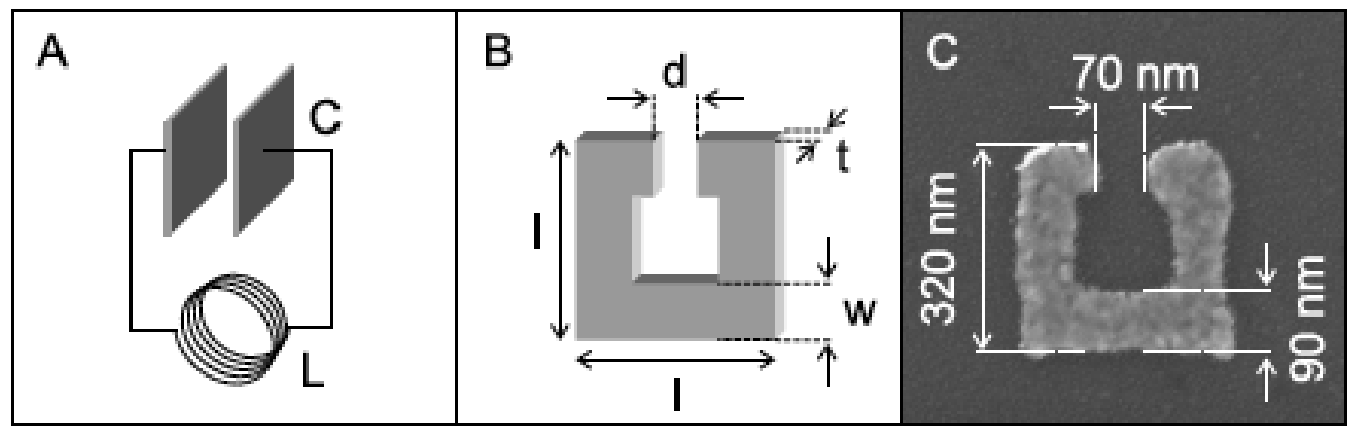

Fig. 9. Illustration of the analogy between a usual LC circuit, A, and a split-ring resonator (SRR), B. The electron micrograph in C shows an actually fabricated structure, a gold SRR $(t=20 \mathrm{~nm})$ on a glass substrate. Taken from Ref. 26. 
Figure 9 shows the analogy of a conventional LC circuit and a metallic SRR on a dielectric substrate. The order of magnitude of the resonance frequency can be easily estimated, by considering the SRR capacitance concentrated in the area of its gap, treating the gap as a parallel plate capacitor, with $C=\varepsilon_{0} \varepsilon_{C} \frac{w t}{d}$ ( $w$ is the width of the metal, $d$ is the width of the gap of the capacitor and $t$ is the metal thickness - see Fig. 6B for these definitions), and using for the inductance the formula for the inductance of a solenoid, i.e. $L=\mu_{0} l^{2} / t$. Thus, $\omega_{L C}=1 / \sqrt{L C}=\frac{c_{0}}{l \sqrt{\varepsilon_{C}}} \sqrt{\frac{d}{w}}$, where $c_{0}$ is the velocity of light in vacuum. The corresponding free space wavelength, $\lambda_{L C}=l(2 \pi) \sqrt{\varepsilon_{C}} \sqrt{w / d}$, is proportional to the size, $l$, of the SRR. In principle, $\lambda_{L C}$ can be much larger than the size of the SRR. This is a fundamental difference between left-handed materials and the other important class of electromagnetic metamaterials, i.e. photonic crystals, where, as was mentioned in the previous section, the frequencies of operation correspond to wavelengths of the same order of magnitude as the size of the unit cell. Since in LHMs the operation wavelength, $\lambda_{L C}$, can be much larger than the size, $a$, of the unit cell, a LHM can be considered very accurately as homogeneous effective medium and described using effective medium theories, which simplify a lot its description and facilitates the physical understanding of its main features.

Indeed, there is a significant amount of theoretical and numerical work, where the homogeneous effective medium assumption was used for the development of a retrieval procedure, which was applied to obtain the effective permittivity, $\mathcal{E}$, and permeability, $\mu$, of a metamaterial from calculated reflection and transmission amplitudes. This procedure confirmed [52-56] that a medium composed of SRRs and 
wires could indeed be characterized by effective $\varepsilon$ and $\mu$ with negative real parts over a finite frequency band, and refractive index with also negative real part.

One can easily obtain a simple expression for the frequency dependence of the effective magnetic permeability $\mu(\omega)$ for a lattice of SRRs, assuming an incident EM field propagating parallel to the SRRs plane, with magnetic field perpendicular to the SRRs plane and electric field parallel to the sides of the SRRs that do not have cuts. Under these conditions, and according to the Kirchhoff loop rule, the selfinduction voltage of the inductance $L, U_{L}$, plus the voltage drop across the capacitance $C, U_{C}$, equals the induced voltage $U_{\text {ind }}$ by the external magnetic flux, i.e.

$$
U_{L}+U_{C}=U_{\text {ind }} \quad \text { or } \quad L \dot{I}+\frac{1}{C} \int I d t=U_{\text {ind }}=-\dot{\phi}
$$

where $\phi$ is the external magnetic flux, $\phi=\mu_{0} l^{2} H, I$ is the current and $H$ is the external magnetic field, $H=H_{0} e^{-i \omega t}$. Taking the time derivative of Eq. (1) we obtain

$$
\ddot{I}+\frac{I}{L C}=\frac{\dot{U}_{i n d}}{L}=+\omega^{2} \frac{\mu_{0} l^{2}}{L} H_{0} e^{-i \omega t}
$$

The obvious solution is $I=I_{0} e^{-i \omega t}$ and we can easily obtain the individual SRR magnetic dipole moment, area current $=l^{2} I$, and the magnetization $M=\left(N_{L C} / V\right) l^{2} I, N_{L C}$ is the number of LC circuits and $V$ is their corresponding volume, i.e. $N_{L C} / V=1 /\left(a_{x y}^{2} a_{z}\right) ; a_{x y} \geq l$ is the lattice constant in the SRR plane and $a_{z} \geq t$ the lattice constant in the direction normal to the SRRs. Finally, using

$$
\begin{gathered}
M=\chi_{m}(\omega) H, \mu(\omega)=1+\chi_{m}(\omega) \text { and } L=\mu_{0} l^{2} / t \text { one obtains that } \\
\mu(\omega)=1+\frac{F \omega^{2}}{\omega_{L C}^{2}-\omega^{2}}=1-\frac{F \omega^{2}}{\omega^{2}-\omega_{L C}^{2}}
\end{gathered}
$$


Apart from the $\omega^{2}$ in the numerator, this represents a Lorenz oscillator resonance for a magnetic atom. Here we have lumped the various parameters into the dimensionless quantity $F=l^{2} t / a_{x y}^{2} a_{z}$, which is less than one. As is done in most cases all the losses and the scattering mechanisms can be lumped into a damping factor $\Gamma_{m}$, added in the denominator of (3). Notice that as $\omega_{L C}<\omega<\omega_{L C} / \sqrt{1-F}, \mu(\omega)<0$.

At the heart of the metamaterials concept is a fundamental physics approach, in which a continuous material conceptually replaces an inhomogeneous collection of scattering objects, described by the relatively simple electromagnetic parameters $\varepsilon$ (the electrical permittivity) and $\mu$ (the magnetic permeability). In general, the material parameters are tensors and are frequency dependent, but nevertheless represent a considerable reduction in complexity for describing wave propagation behavior.

It has been found that the averaged response of artificially structured metamaterials follows well known forms of response that occur in conventional materials. A common means of describing material properties, for example, is in terms of the Drude-Lorentz model, in which the details of a material are replaced conceptually by a collection of harmonically bound charges - either electric or fictitious magnetic. The bound charges are displaced by incident (electric or magnetic) fields, resulting in a responding polarization in the medium. If the polarization is linearly related to the applied fields, then Maxwell's equation combined with the oscillator model yields the well-known effective material parameters:

$$
\varepsilon(\omega)=1-\frac{\omega_{p e}^{2}}{\omega^{2}-\omega_{0 e}^{2}+i \Gamma_{e} \omega} \quad \mu(\omega)=1-\frac{\omega_{p m}^{2}}{\omega^{2}-\omega_{0 m}^{2}+i \Gamma_{m} \omega}
$$


These are the standard Drude-Lorentz forms for the permittivity and permeability. Their form stems from the universal resonant response of an harmonic oscillator to an external frequency dependent perturbation. Note that three parameters enter: the plasma frequency, $\omega_{p}$, the resonant frequency, $\omega_{0}$, and a damping factor, $\Gamma$. These parameters are indexed with an " $\mathrm{e}$ " for electric or an " $\mathrm{m}$ " for magnetic response. The Drude-Lorentz form correctly describes the electromagnetic response of materials over frequencies that range from microwaves to optical or UV, thus providing a convenient language uniting the description of materials over all frequency ranges. Free electron contribution in metals correspond to $\omega_{0 e}=0$. We point out in passing that the concept of resonance is also crucial for the existence of photonic crystals. The difference from the LHMs is that in photonic crystals the resonance is purely geometrical and occurs when the wavelength inside the material is comparable to the period of the structure. In contrast, the LHMs could have characteristic structural lengths much smaller that the wavelength permitting thus their effective homogeneous description. This property of LHMs could be used in the miniaturization of devices.

Metamaterials based on strong artificial resonant elements can also be described quite efficiently with formulas similar to the Drude-Lorentz forms. In fact, two types of artificial elements have served as the basic building blocks for a wide variety of metamaterials. These structures are the metallic wire structure, which provides a predominantly free electron response to electromagnetic fields, and the split ring resonator structure, which provides a predominantly magnetic response to electromagnetic fields.

Materials with $\varepsilon$ negative are well known, and have been investigated for many years $[7,8]$. In naturally occurring materials, the resonances that give rise to the Drude-Lorentz forms occur within generally restricted frequency ranges. Electric resonances, for example, tend to occur in the high $\mathrm{THz}$ frequencies or much higher, and result from phonon modes, plasma like oscillations of the conduction electrons, or other fundamental processes. Magnetic resonances generally occur in inherently magnetic materials, 
associated with such processes as ferromagnetic or antiferromagnetic resonance. These resonances tend to die out in the higher $\mathrm{GHz}$ frequencies, and are absent in all but a few specialized systems at $\mathrm{THz}$ frequencies

While conventional material response appears to be restricted, this is not a fundamental limitation, and thus metamaterials can be designed that have either electric or magnetic resonances where there are no equivalent existing materials. Recent work, including our contributions, has demonstrated that electric and magnetic resonances can be situated at any frequency, up to $\mathrm{THz}$ frequencies, in metamaterial structures. In particular, by combining electric and magnetic structures, it is possible to arrive at a material with a frequency band over which both $\varepsilon$ and $\mu$ are simultaneously negative. The refractive index, $n$, for such a material, determined by taking the square root of the product $\varepsilon \mu$, is real, indicating the material is transparent to radiation. However, it has been shown that the correct choice for the sign of the square root is negative when both $\varepsilon$ and $\mu$ are negative. Thus, materials for which $\varepsilon$ and $\mu$ are both negative can be also characterized as negative index materials (NIMs).

\section{Saturation of the magnetic response of SRR at optical frequencies}

There is sustained effort in the community to push the operation frequency of the metamaterials deeper and deeper in the THz region to reach ultimately optical frequencies. This is so important because there are no natural materials that have magnetic properties at such high frequencies. At low frequencies, up to several $\mathrm{THz}$, the magnetic resonance frequency scales reciprocally with the structural size. At high frequencies, however, this linear scaling breaks down, as was shown in Ref. 57. The reason is the following: There are in general two velocity dependent contributions to the energy of a metallic wire in which an electric current, $I=e w t n_{e} v_{e}$, is flowing ( $w t$ is the cross-section of the wire - see Fig. 9B,$- n_{e}$ is 
the concentration of free electrons and $v_{e}$ is their mean velocity). One is the magnetic energy, $L_{m} I^{2} / 2$, and the other is the kinetic energy of the free electrons, $E_{k}=N_{e} m_{e} v_{e}^{2} / 2=V n_{e} m_{e} v_{e}^{2} / 2$, where the volume, $V$, of the wire is $V=w t l^{\prime}$, with $l^{\prime}=4(l-w)-d$ (see Fig. 9B), being the length of the wire. To compare these two contributions we can re-express the kinetic energy in terms of $I$ instead of $v_{e}: E_{k}=L_{e} I^{2} / 2$, where $L_{e}=l^{\prime} m_{e} / w t e^{2} n_{e}=\left(l^{\prime} / w t\right)\left(1 / \omega_{p}^{2} \varepsilon_{0}\right)$. Thus, for a wire making a ring like the one of Fig. 9B, when all the sizes $(w, t, d, l)$ scale proportional to the unit cell size, $a$, the effective inductance $L_{e}$, corresponding to $E_{k}$, scales inversely proportional to $a$, in contrast to the magnetic inductance, $L_{m}$, which is proportional to $a$. The ratio $L_{m} / L_{e}$ is of the order of $10 w t / \lambda_{p}^{2}$, with the typical value of $\lambda_{p}$ being around $100 \mathrm{~nm}$. Thus, for $\sqrt{w t}$ considerably larger than $100 \mathrm{~nm}$ the kinetic energy, $E_{k}$, of the electrons is negligible in comparison with the magnetic energy; but for $\sqrt{w t}$ smaller than $100 \mathrm{~nm} \quad E_{k}$ becomes appreciable and may dominate as $\sqrt{w t}$ becomes smaller and smaller.

The capacitance $C$ of the SRR scales also proportional to the size of the SRR, so the magnetic resonance frequency has the following size dependence

$$
\omega_{L C}=\frac{1}{\sqrt{\left(L_{m}+L_{e}\right) C}} \propto \frac{1}{\sqrt{\text { size } e^{2}+\text { const }} .}
$$

For very large structures, the resonance frequency is inversely proportional to the size of the SRR, while for small structures $\omega_{L C}$ approaches a constant. To get a rough estimate of the saturation value of $\omega_{L C}$ we take into account that the capacitance $C$ is given by $C=\varepsilon_{0} w t / d$. By using the expression of the metal plasma frequency, $\omega_{p}^{2}=n_{e} e^{2} / \varepsilon_{0} m_{e}$, we obtain that the saturation magnetic resonance frequency is of the order of 


$$
\omega_{L C}^{\max }=\frac{1}{\sqrt{L_{e} C}} \approx \omega_{p} \sqrt{\frac{d}{4 l}}
$$
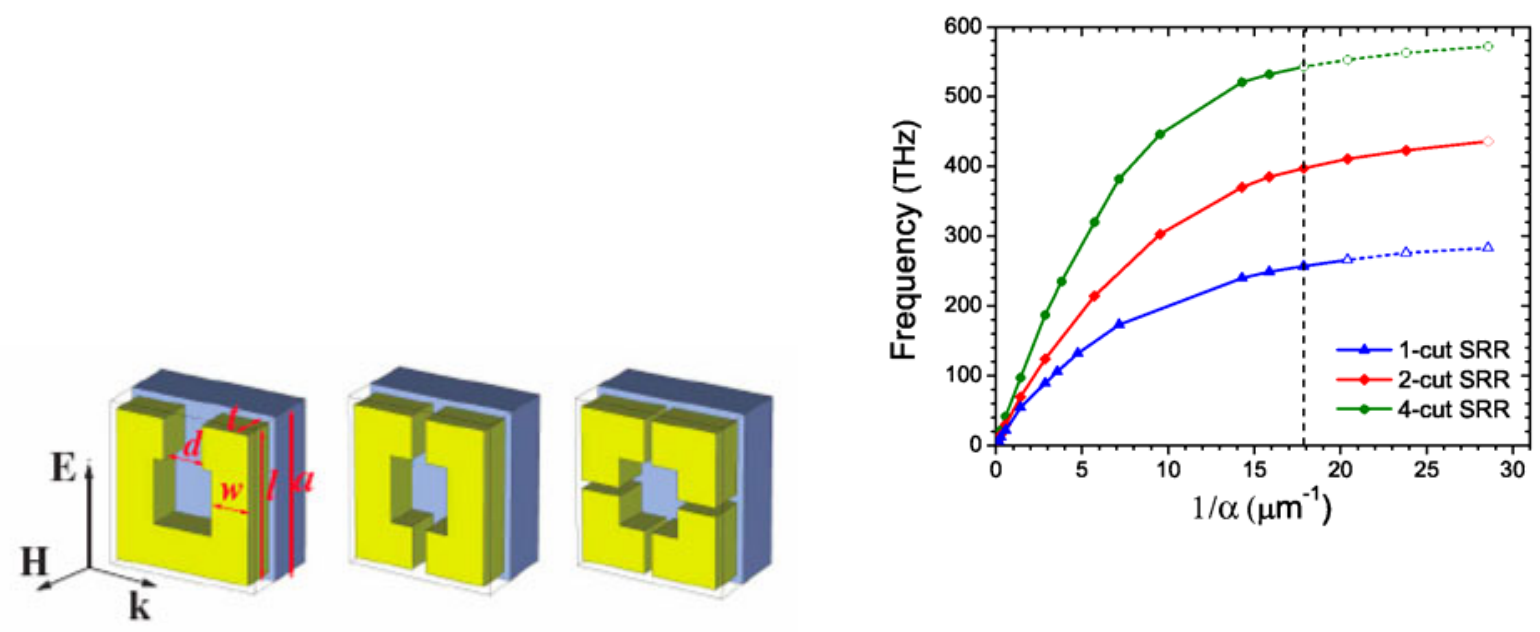

Fig. 10. The geometries of the 1-, 2-, and 4- cut single-ring

Fig. 11. The scaling of the simulated magnetic SRRs are shown; the unit cell has the dimensions $a \quad a$ in the SRR plane and 0:614a perpendicular to it. The SRR is made of aluminum, simulated using a Drude-model permittivity $\left(f_{p}=\omega_{p} / 2 \pi=3570 \mathrm{THz}, f_{\tau}=19.4 \mathrm{THz}\right)$. scaling is linear, $f_{m} \propto 1 / a$. The maximum attainable frequency is strongly enhanced with the number of cuts in the SRR ring. The hollow symbols as well as the vertical line at $1 / a=17.9 \mu \mathrm{m}^{-1}$ indicate that no $\mu<0$ is reached anymore.

This saturation frequency is further reduced by the dielectric environment and by the skin effect [57], 
which have been neglected in our rough estimate. In Ref. 57, the limits of the resonant magnetic response for single-ring multicut SRR designs, shown in Fig. 10, have been investigated up to optical frequencies. It was shown (see also Fig. 11) that the breakdown of linear scaling due to the free electron kinetic energy occurs for frequencies above $100 \mathrm{THz}$. Well above the linear scaling regime, the resonance frequency saturates, while the amplitude of the resonant permeability decreases, ultimately ceasing to reach negative value. The highest resonance frequency at which $\mu<0$ increases with the number of cuts in the SRR. The highest magnetic resonance frequency is obtained for 4 or even more cuts.

We would like to stress that the 4-cut single ring SRR design is favorable for higher dimensional LHMs not only for its highest attainable magnetic resonance frequency but also for its inherent symmetry [11, 58, 59]. The general problem with the single gap SRRs is the inherent asymmetry of the resulting lattice of SRRs. As was discussed in Ref. 11, this will lead to the undesirable excitation of the magnetic resonance by the electric field. To avoid this coupling, more symmetric SRRs should be used. Koschny, Zhang and Soukoulis [59] proposed a three-dimensional isotropic LHM design, shown in Fig. 12, based on single-ring 4-gap SRRs, that allows left-handed behavior for any direction of propagation and any polarization of the electromagnetic wave. Using numerical transfer matrix simulations, they verified the isotropic transmission properties of the proposed structure. Their data show excellent agreement with results expected for a homogeneous slab with the corresponding negative $\varepsilon$ and $\mu$. No 3D isotropic LHM has been fabricated so far, and it is a challenge to be build even at microwaves frequencies.
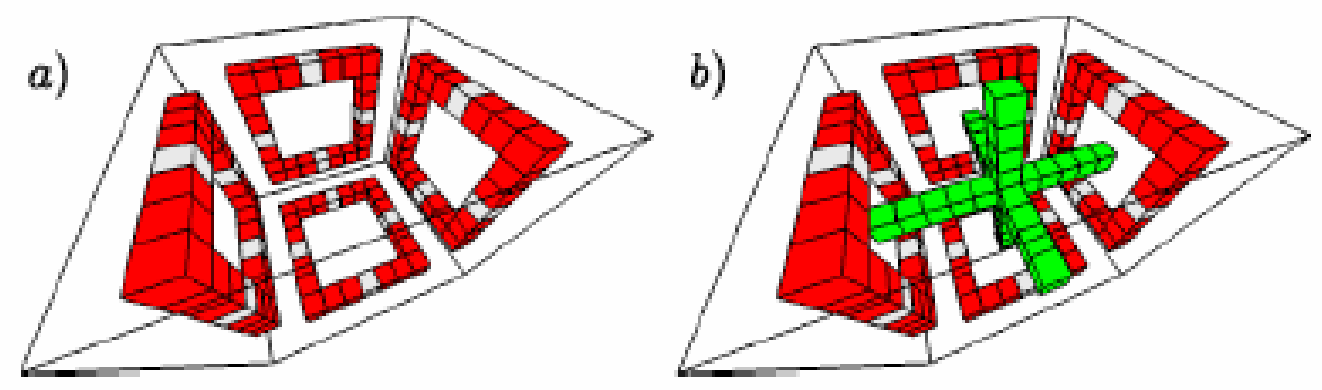
Fig. 12. The design of a fully symmetric unit cell of an isotropic SRR, (a), and a left-handed metamaterial based on this symmetric SRR design, (b). The interfaces are parallel to the left and right SRR. The metal of the four-gap SRR (red/dark gray) and the continuous wires (green/medium gray) is silver using a Drude model permittivity around 1 THz. The SRR gaps are filled with a high-constant dielectric slight gray with a relative permittivity $\varepsilon_{\text {gap }}=300$ to lower the magnetic-resonance frequency.

\section{Negative index materials using simple short wire pairs}

All NIM implementations to date have utilized the topology proposed by Pendry, consisting of SRRs and continuous wires. A lot of groups were able to fabricate [2,3,9-24] NIMs with an index of refraction $n=-$ 1 with losses of less than $1 \mathrm{~dB} / \mathrm{cm}$, in the GHz regime. Recently different groups observed indirectly [25$29,41]$ negative $\mu$ at the $\mathrm{THz}$ region. In most of the THz experiments $[25,26,41]$ only one layer of SRRs were fabricated on a substrate and the transmission, $T$, was measured only for propagation perpendicular to the plane of the SRRs, exploiting the coupling of the electric field to the magnetic resonance of the SRR via asymmetry [11]. This way it is not possible to drive the magnetic permeability negative. Also, no negative $n$ with small imaginary part has been observed yet at the $\mathrm{THz}$ region. One reason is that is very difficult to measure with the existing topology of SRRs and continuous wires both the transmission, $T$, and reflection, $R$, along the direction parallel to the plane of the SRRs. So there is a need for alternative, improved and simplified designs that can be easily fabricated and experimentally characterized, especially in the infrared and optical regions of the spectrum. Such a design is offered by pairs of finite in length wires (short-wire-pair).

A short-wire-pair can behaves like an SRR, exhibiting a magnetic resonance followed by a negative permeability regime. Moreover, short-wire-pairs can give simultaneously a negative $\varepsilon$ in the same frequency range, and therefore a negative $n$, without the need for additional continuous wires [38-40]. 
Recent experiments [29] have not shown though evidence of negative $n$ at THz frequencies in the short wires-pair cases that were studied. This is in contrast with the claims [42] that one can get negative $n$ at $\mathrm{THz}$ frequencies. The negative $n$ obtained [41-43] at THz frequencies is most probably due to the large imaginary parts of $\varepsilon$ and $\mu$ [44].

Very recent unpublished work $[60,61]$ introduced new designs of short-wire-pair based metallic structures to obtain negative index of refraction in the microwaves regime. The basic structure of a single unit cell of this NIM was build from H-shaped wires and is as shown in Fig. 13: The conventional SRR is replaced by a pair of short parallel wires, which provide both negative magnetic and negative electric response; the continuous wires are not necessary. The short-wire-pair consists of a pair of metal patches separated by a dielectric spacer of thickness $t_{s}$. For an electromagnetic wave incident with a wave vector and field polarization as shown in Fig. 13, the short-wire-pair will acquire not only a magnetic resonance resulting in a negative $\mu[29,42,61]$ but also an electric resonance with a negative $\varepsilon$ simultaneously. The magnetic resonance originates from the anti-parallel current in the wire pair with opposite sign charge accumulating at the corresponding ends; the electric resonance is due to the excitation of parallel current in the wire pair with same sign charge accumulating at the corresponding ends of both wires. Repeating this basic structure periodically in the $\mathrm{x}-, \mathrm{y}-$, and z-directions would result in a NIM structure. 

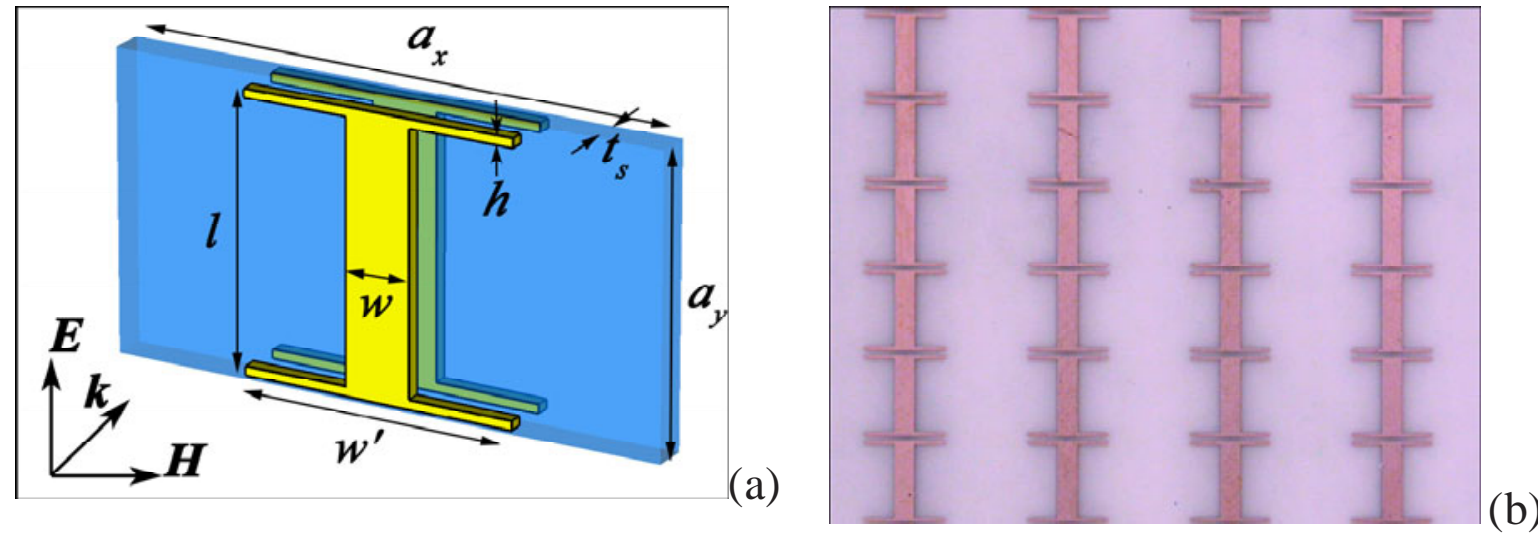

Fig. 13: (a) Schematic representation of one unit cell of the wire-pair structure. (b) Photograph of fabricated microwave-scale wire-pair sample.

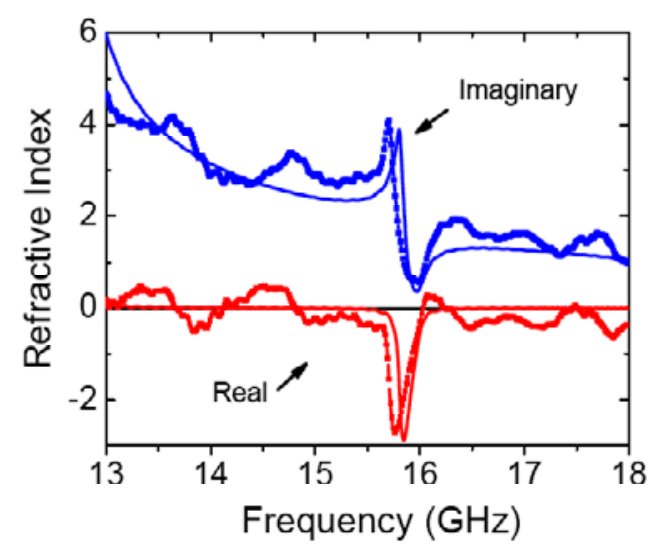

Fig. 14. Extracted refractive index $n$ of a periodic array of wire-pair unit cells, using the simulated (solid curves) and measured (dotted curves) transmission and reflection data. The red and blue curves show the real part of $n$ and imaginary part of $n$ respectively

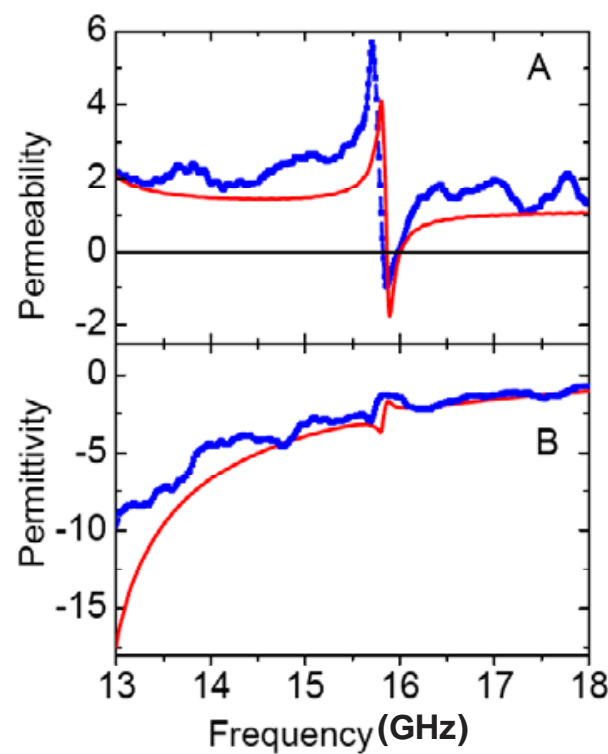

Fig. 15. Extracted permittivity $\varepsilon$ (A) and permeability (B) $\mu$ of a periodic array of wire-pair unit cells, using the simulated (red solid curves) and measured (blue dotted curves) transmission and reflection data. 
Transmission and reflection properties of a single-layer structure were measured over the frequency range of 13-18 GHz using a network analyzer (HP 8510) and a pair of standard gain horn antennas serving as source and receiver. In the transmission measurements, the microwaves were incident normal to the sample surface. This is a tremendous simplification relatively to the conventional SRRs and wires where the incident electromagnetic waves have to propagate parallel to the sample surface. With the conventional orientation of the SRRs, it is almost impossible to do these type of measurements at the $\mathrm{THz}$ region, since only single-layer samples are usually fabricated $[25,26]$. In both measurements, the electric field of the incident wave was polarized parallel to the long dimension of the wires. (For perpendicular polarization the transmission at the resonance regime was nearly $100 \%$, independently of the frequency, and the reflection was essentially zero.) Using the transmission and reflection results from a single layer, we can extract the effective refractive index that would result if a periodic multi-layer sample were built using the single-layer structure as a building block. The details of the numerical retrieval procedure have been described in detail elsewhere [45-50]. The extracted refractive index is shown in Fig. 14 and the extracted permittivity and permeability are shown in Fig. 15. The plots show the real part of the permittivity (see Fig. 15A) is negative over most of the measured range. The real part of the permeability is negative over a band near $16 \mathrm{GHz}$ for the simulation and the experiment. The extracted real part of the refractive index is negative [44] over a narrow band at $16 \mathrm{GHz}$. The ratio of the imaginary part of $n$ to the real part of $n$ is $1 / 3$, which means that we have left-handed propagation with $\mathcal{E}, \mu$ and $n$ negative.

Our preliminary numerical results show that if our structure is scaled down by a factor of 200 , it will give a negative index of refraction at $\mathrm{THz}$ frequencies, with both $\varepsilon$ and $\mu$ are negative. These results show clearly the viability of using short wire pairs to build negative-index materials, if combined with 
additional continuous wires. It is likely that modifications of the basic structure studied here may improve or alter the NIM properties. Also, wire-pair arrangements with significantly different geometries may lead to negative-index materials. The relative ease of fabricating wire-pair structures may hasten the development of NIMs working at optical wavelengths.

\section{Concluding remarks}

NIMs have rapidly achieved widespread recognition, as they allow previously unavailable solutions of Maxwell's equations. As such, NIMs represent a striking example of the utility of metamaterials. Yet, although remarkable physical phenomena have been predicted for NIMs, including evanescent wave refocusing (leading to "perfect lensing"), nearly aberration free lenses, reversed Doppler shifts and reversed Cerenkov radiation, the limitations of negative materials must be kept in mind. For example, it has been suggested that a surface with $\varepsilon=\mu=-1$ can be reflectionless. This statement, however, is only true in a steady-state sense; if a wave front from free space impinges on such a surface, reflections associated with transients will, in fact, occur until the steady state solution is reached.

Efforts over the past several years, including ours, have been instrumental in proving that negative index metamaterials can be designed, fabricated and characterized. Negative refraction, in steady state experiments, has now been demonstrated many times. Experiments showing image resolution beyond the diffraction limit via a negative index slab have been published. Thus the work of several groups in the last four years has placed negative refractive index on solid ground: We are now in a position to move forward and further develop the materials and methods that will make these novel materials useful.

In particular we believe that the uniqueness and novelty of LHMs or NIMs are:

(a) The ability to match the vacuum impedance (zero reflectance!) is a unique property of NIMs with 
many applications.

(b) Also, the possibility of creating patterns that allow for coupling with the magnetic component of an electromagnetic field without the presence of any magnetic material is a new capability of fundamental importance, especially in the THz region where no magnetic natural resonance exists.

(c) Miniaturization of devices and components, such as antennas and waveguide structures, especially at long $\lambda$, is very important, due to potential system weight and size savings. NIMs provide a platform for a revolutionary change in the design of sub- $\lambda$ devices.

(d) Finally, the capability of having a negative index of refraction opens up the possibility of new applications in optics and communications.

\section{Acknowledgements}

It is a pleasure to acknowledge stimulating discussions on scientific issues discussed in this review with many students, post-docs and colleagues, in particular, Th. Koschny, Lei Zhang, Jiangfeng Zhou, M. Wegener, S. Linden, and E. Ozbay. We gratefully acknowledge the support of Ames Laboratory, which is operated by Iowa State University under contract No. W-7405-Eng-82, of EU projects DALHM, METAMORHOSE, PHOREMOST, of DARPA (Contract No. HR0011-05-C-0068), and of the Greek Ministry of Education (PYTHAGORAS project). 


\section{References}

1. V. G. Veselago, Soviet Physics, USPEKHI 10, 509 (1968).

2. D. R. Smith, W. J. Padilla, D. C. Vier, S. C. Nemat-Nasser, \& S. Schultz, Phys. Rev. Lett. 84, 4184-4187 (2000).

3. R. A. Shelby, D. R. Smith, \& S. Schultz, Science 292, 77-79 (2001).

4. J. B. Pendry, A. Holden, D. Robbins and W. Stewart, IEEE Trans. Microwave Theory Tech. 47, 2075 (1999); J.B. Pendry, A.T. Holden, W.J. Stewart and I. Youngs, Phys. Rev. Lett., 25, 4773 (1996); J.B. Pendry, A. J. Holden, D. J. Robbins, and W. J. Stewart, J. Phys. Cond. Matt., 10, 4785 (1998).

5. M. Wiltshire, J. B. Pendry, I. R. Young, D. J. Larkman, D. J. Gilderdale and J. V. Hajnal, Science 291848 (2001).

6. J. B. Pendry, Phys. Rev. Lett. 85, 3966 (2000).

7. For a recent review see: D. R. Smith, J. B. Pendry \& M. Wiltshire, Science, 305, 788(2004); D. R. Smith, J. B. Pendry, Physics Today, p37 (June, 2004).

8. For a recent review see: S. A. Ramakrishna, Rep. Prog. Phys. 68, 449 (2005)

9. R. A. Shelby, D. R. Smith, S. C. Nemat-Nasser and S. Schultz, Appl. Phys. Lett., 78, 4 (2001)

10. M. Ayindir, K. Aydin, E. Ozbay, P. Markos, \& C. M. Soukoulis, Appl. Phys. Lett. 81, 120-122 (2002).

11. N. Katsarakis, T. Koschny, M. Kafesaki, E. N. Economou and C. M. Soukoulis, Appl. Phys. Lett. 84, 2943 (2004).

12. Lei Zhang, G. Tuttle and C. M. Soukoulis, Photonic and Nanostructures 2, 155 (2004).

13. N. Katsarakis, T. Koschny, M. Kafesaki, E. N. Economou, E. Ozbay and C. M. Soukoulis, Phys. Rev. B. 70, 201101(R) (2004).

14. K. Aydin, K. Guven, Lei Zhang, M. Kafesaki, C. M. Soukoulis, \& E. Ozbay, Optics Letters, 29, 2623 (2004).

15. K. Aydin, K. Guven, N. Katsarakis, C. M. Soukoulis, and E. Ozbay, Optics Express 24, 5896 (2004).

16. K. Guven, K. Aydin, K.B. Alici, C.M. Soukoulis, and E. Ozbay, Phys. Rev. B 70, 205125 (2004).

17. R. Moussa, S. Foteinopoulou, Lei Zhang, G. Tuttle, K. Guven, E. Ozbay and C. M. Soukoulis, Phys. Rev. B 71, 085106 (2005).

18. K. Aydin, K. Guven, C. M. Soukoulis, and E. Ozbay, Appl. Phys. Lett. 86, 124102 (2005). 
19. C. G. Parazzoli, R. B. Greegor, K. Li, B. E. C. Koltenbach \& M. Tanielian, Phys. Rev. Lett. 90, 107401 (2003).

20. K. Li, S. J. Mclean, R. B. Greegor, C. G. Parazzoli \& M. Tanielian, Appl. Phys. Lett. 82, 2535 (2003).

21. A. A. Houck, J. B. Brook and I. L. Chuang, Phys. Rev. Lett. 90, 137401 (2003).

22. E. Cubukcu, K. Aydin, E. Ozbay, S. Foteinopoulou and C. M. Soukoulis, Nature 423, 604 (2003).

23. E. Cubukcu, K. Aydin, E. Ozbay, S. Foteinopoulou and C. M. Soukoulis, Phys. Rev. Lett. 91, 207401 (2003).

24. P. V. Parini et. al. Nature 426, 404 (2003); P. V. Parimi et. al. Phys. Rev. Lett. 92, 127401 (2004); P. Vodo et. al. Appl. Phys. Lett. 85, 1858 (2004); ibid Appl. Phys. Lett. 86, 201108 (2005).

25. T. J. Yen, W. J. Padilla, N. Fang, D. C. Vier, D. R. Smith, J. B. Pendry, D. N. Basov, \& X. Zhang, Science 303, 1494-1496 (2004).

26. S. Linden, C. Enkirch, M. Wegener, J. Zhou, T. Koschny \& C, M, Soukoulis, , Science 306, 1351 (2004).

27. N. Katsarakis, G. Konstantinidis, A. Kostopoulos, R. S. Penciu, T. F. Gundogdu, Th Koschny, M. Kafesaki, E. N. Economou, and C. M. Soukoulis, Optics Letters 30, 1348 (2005).

28. C. Enkrich, S. Linden, M. Wegener, S. Burger, L. Zswchiedrich, F. Schmidt, J. Zhou, T. Koschny and C. M. Soukoulis, Phys. Rev. Lett. 95, 203901 (2005).

29. C. Enkrich, F. Perez-Willard, D. Gerthsen J. Zhou, T. Koschny, C. M. Soukoulis, M. Wegener and S. Linden, Advanced Materials 17, 2543 (2005); G. Dolling et. al. Opt. Lett. 30, 3198 (2005).

30. N. Fang et. al. Science 308, 534 (2005); D. R. Smith, Science, 308, 502 (2005).

31. M. Notomi, Phys. Rev. B 62, 10696 (2000)

32. C. Luo et. al. Phys. Rev. B 65, 201104 (2002); ibid Phys. Rev. B 68, 045115 (2003).

33. S. Foteinopoulou, E. N. Economou and C. M. Soukoulis, Phys. Rev. Lett. 90, 107402 (2003).

34. S. Foteinopoulou, and C. M. Soukoulis, Phys. Rev. B 67, 235117(2003); ibid Phys. Rev. B 72, 165112 (2005).

35. C. G. Parazolli et.al., Appl. Phys. Lett. 84, 3232 (2004).

36. A. Berrier et. al., Phys. Rev. Lett. 93, 073902 (2004)

37. E. Schonbrum et. al. IEEE Photon. Tecnolog. Lett. 17, 1196 (2005).

38. A. N. Lagarkov and A. K. Sarychev, Phys. Rev. B 53, 6318 (1996)

39. L. V. Panina, A. N. Grigorenko and D. P. Makhnorskiy, Phys. Rev. B 66, 155411 (2002)

40. V. A. Podolskiy, A. K. Sarychev, and V. M. Shalaev, J. Nonlinear Opt. Phys. Matt. 11, 65 (2002); V. A. Podolskiy, A. K. Sarychev and V. M. Shalaev, Opt. Express 11, 735 (2003) 
41. S. Zhang et. al, Phys. Rev. Lett. 94, 037402 (2005); S. Zhang et. al. Phys. Rev. Lett. 95, 137404 (2005)

42. V. M. Shalaev, et. al. Opt. Lett. 30, 3356 (2005)

43. A. N. Grigorenko et. al. Nature 438, 335 (2005)

44. In lossy materials is possible to have the real part $n$ to be negative, without having the real parts of e and $\mathrm{m}$ simultaneously negative. This is the case of the recent work of S. Zhang et. al. Phys. Rev. Lett. 95,137404 (2005). This can happen if the imaginary parts of $\varepsilon$ and $\mu$ are sufficiently large, because in a lossy material $n=n^{\prime}+i n^{\prime \prime}$, and we also have that $n=\varepsilon z$ and $z=\sqrt{\mu / \varepsilon}$. After some algebra we obtain that $n^{\prime}=\varepsilon^{\prime} z^{\prime}-\varepsilon^{\prime \prime} z^{\prime \prime}$ and $z=\sqrt{\left(\mu^{\prime} \mathcal{E}^{\prime}+\mu^{\prime \prime} \varepsilon^{\prime \prime}\right) / \varepsilon^{2}+i\left(\mu^{\prime \prime} \mathcal{E}^{\prime}-\mu^{\prime} \mathcal{E}^{\prime \prime}\right) / \varepsilon^{2}}$, so it's possible to have $n^{\prime}<0$ provided that $\mathcal{E}^{\prime \prime} z^{\prime \prime}>\mathcal{E}^{\prime} z^{\prime}$. In this scenario however, the imaginary parts lead to dominant losses such that we have a transmission gap with some negative phase shift rather than LH transmission (with some losses). This type of negative $n$ should not be considered LH behavior. In our experiments [54, 55], although we have considerable imaginary parts, the behavior is still dominated by the negative real part of $n$ at the highfrequency side where we find the LH behavior.

45. M. Sigalas, C. M. Soukoulis, C. T. Chan and K. M. Ho, Phys. Rev. B 49, 11080 (1994).

46. S. O'Brien and J. B. Pendry, J. Phys: Condens. Matt. 14, 4035 (2002).

47. K. C. Huang, M. L. Povinelli and J. D. Joannopoulos, Appl. Phys. Lett. 85, 543 (2004).

48. G. Shvets, Phys. Rev. B 67, 035109 (2003).

49. A. A. Zharov, I. V. Shadrivov, Yu. S. Kivshar, Phys. Rev. Lett.91, 037401 (2003).

50. I. V. Shardriviv et. al. Phys. Rev. E. 69, 016617 (2004); M. W. Feise, I. V. Shadrivov and Yu. S. Kivshar, Appl. Phys.. Lett. 85, 1451 (2005).

51. T. Koschny, M. Kafesaki, E. N. Economou, and C. M. Soukoulis, Phys. Rev. Lett. 93, 107402 (2004).

52. D. R. Smith, S. Schultz, P. Markos and C. M. Soukoulis, Phys. Rev. B 65, 195104 (2002).

53. T. Koschny, P. Markos, D. R. Smith and C. M. Soukoulis, Phy. Rev. E 68, 065602(R) (2003).

54. T. Koschny, P. Markos, D. R. Smith and C. M. Soukoulis, Phys. Rev. E 70, 048603 (2004).

55. D. R. Smith, D. C. Vier, Th. Koschny, and C. M. Soukoulis, Phys. Rev. E 71, 121103 (2005).

56. Th. Koschny, P. Markos, E. N. Economou, D. R. Smith, D. C. Vier, and C. M. Soukoulis, Phys. Rev. B 71, 245105 (2005). 
57. J. Zhou, Th. Koschny, M. Kafesaki, E. N. Economou, J. B. Pendry and C. M. Soukoulis, Phys. Rev. Lett. 95, 223902 (2005).

58. .M. Kafesaki et. al. J. Opt. A: Pure Appl. Opt. 7, S12 (2005).

59. Th. Koschny, Lei Zhang, and C. M. Soukoulis, Phys. Rev. B 71, 036617 (2005).

60. J. Zhou, Lei Zhang, G. Tuttle, Th. Koschny and C. M. Soukoulis, Phys. Rev. B 73, 041101 (R) (2006).

61. J. Zhou, Lei Zhang, G. Tuttle, Th. Koschny and C. M. Soukoulis, Appl. Phys. Lett. (submitted). 


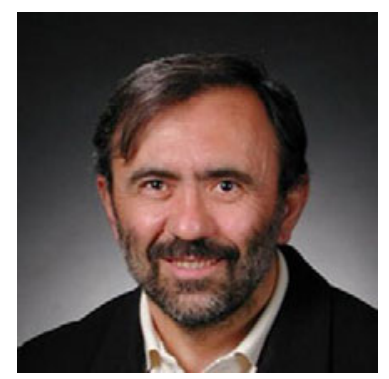

Costas Soukoulis received his B.S. in Physics from Univ. of Athens in 1974. He obtained his doctoral degree in Physics from the Univ. of Chicago in 1978. From 1978 to 1981 he was visiting Assistant Professor at the Physics Dept. at Univ. of Virginia. He spent 3 years (1981-84) at Exxon Research and Engineering Co. and since 1984 has been at Iowa State Univ. (ISU) and Ames Laboratory. He is currently a Distinguished Professor of Physics at ISU. He has been an associated faculty member of FORTH since 1984 and since 2001 is also a Professor (part-time) at Dept. of Materials Science and Engineering at Univ. of Crete. He has approximately 300 publications, more than 70 invited lectures at national and international conferences, and about 100 invited talks at institutions. He has more than 7000 citations and 2 patents for photonic band gap materials. Has obtained several grants to support his research from DOE, NSF, DARPA, NATO, EPRI, and European Community. Prof. Soukoulis is Fellow of the American Physical Society, Optical Society of America, and American Association for the Advancement of Science. He received the ISU Outstanding Achievement in Research in 2001, and the senior Humboldt Research Award in 2002; he shared the Descartes award for collaborative research on left-handed materials in 2005. He is the senior Editor of the new Journal "Photonic Nanostructures: Fundamentals and Applications". 
